\title{
UVB EXPOSURE INDUCED ACCUMULATION OF PHENOLICS AND RESVERATROL AND ENHANCED ANTIOXIDANT ACTIVITIES IN PEANUT SPROUTS
}

\author{
Van Lam Nguyen ${ }^{1 凶}$, Thi Ninh Dinh ${ }^{1}$, Huy Bac Nguyen $^{1}$, Thi My Anh Nguyen ${ }^{1}$, Thi Yen Vu ${ }^{1}$ and \\ Thi Phuong Lam Thieu', Vinh Hoang Nguyen ${ }^{2}$, Thi Ngoc Ha Lai ${ }^{1}$ \\ ${ }^{1}$ Department of Biochemistry and Food Biotechnology, Faculty of Food Science and Technology, Vietnam \\ National University of Agriculture, Hanoi, Vietnam \\ ${ }^{2}$ Department of Food Safety and Quality Management, Faculty of Food Science and Technology, Vietnam \\ National University of Agriculture, Hanoi, Vietnam \\ ${ }^{\bowtie}$ lamvan.nguyen@yahoo.com
}

https://doi.org/10.34302/crpifst/2020.12.1.9

Article history:

Received:

Accepted:

25 May 2019

29 February 2020

\section{Keywords:}

Antioxidants;

Arachis hypogaea L;

Resveratrol;

Phenolics;

$U V B$.

\begin{abstract}
Resveratrol is a phenolic compound which naturally presents in peanuts (Arachis hypogaea L.) but with low amount. Resveratrol and phenolic compounds are induced in plants by UVB exposure. This study aimed to investigate the accumulation of phenolics and resveratrol and antioxidants in sprouts of three peanut cultivars, Lac sen, L14 and L27. Peanut seeds were germinated under control and UVB exposure (one hour every day at 9 am). Sprouts were harvested at three stages: 1, 3 and 5 days (D1, D3 and D5). Total phenolic (TP) and resveratrol contents and antioxidant activity were measured using Folin-Ciocalteu, HLPC coupled with UV detector and DPPH methods, respectively. The study found both germination time and UVB exposure induced the accumulation of phenolics and resveratrol and increased the antioxidant activity. Compared to D1 sprouts, D5 sprouts had significant increases in TP and resveratrol contents and the antioxidant activity by $42.31 \%$, $508.75 \%$ and $77.91 \%$, respectively, while UVB exposure enhanced TP and resveratrol contents and the antioxidant activity by $11.11 \%, 62.81 \%$ and 26.17\%. Resveratrol content ranged from $5.57 \mu \mathrm{g} / \mathrm{g}$ DW in L27 sprouts at D1 under control conditions to $110.16 \mu \mathrm{g} / \mathrm{g}$ DW in Lac sen sprouts at D5 under UVB exposure. UVB exposure induced the accumulation of phenolics, particularly resveratrol in peanut sprouts, suggesting this is a potential approach to produce functional foods from peanut sprouts.
\end{abstract}

\section{Introduction}

Peanut (Arachis hypogaea L.) is one of the most important species belonging to Leguminosae family, which is the third largest family of higher plants. It is an industrial crop with high economic value in agricultural sector not only because of its oil but also of its sources of proteins, minerals and vitamins (Krishna et al., 2015). Furthermore, peanut is a source of diverse bioactive substances such as stilbene, flavonoid, phenolic acid, and phytosterols which are beneficial for human health. They can function as antioxidants, and can be involved in activating liver detoxification enzymes, blocking bacterial activity or virus toxins, inhibiting cholesterol absorption and reducing platelet aggregation (Pennington, 2002). 
Resveratrol, a phenolic compound belonging to the stilbene group, has been a bioactive substance of interest over the past decade due to its potential benefits for human health. Resveratrol was first identified in the roots of white hellebore (Veratrum grandiflorum O Loes) in 1940 (Aggarwal et al., 2004). This substance was then found in grapes, red wine and peanuts (King et al., 2006). Studies show that resveratrol reduces the risk of cardiovascular disease, Alzheimer's disease, inhibits cancer growth and slows the aging process (Sales and Resurreccion, 2014; Hasan et al., 2013). Recently, Ha et al. (2015) revealed supplementary of peanut sprout extracts reduced abdominal fat and enhanced health indices in obese women.

Resveratrol naturally exists in peanut seeds but its content is low; therefore, the investigation of methods to increase resveratrol content in peanuts has recently been of interest. Germination is considered an approach to increase the content of bioactive substances such as phenolics in some legumes (Tang et al., 2014; Lin and Lai, 2006) and GABA in rice (Zhang et al., 2014). Some studies on peanuts found that germination enhanced resveratrol contents in peanut sprouts (Limmongkon et al., 2017; Wang et al., 2005). Soaking temperature, germination temperature and germination time also have effects on resveratrol content; a study by $\mathrm{Yu}$ et al. (2016) indicated that peanut seeds soaked at $35^{\circ} \mathrm{C}$ for 6 hours resulted in the highest resveratrol content and the best germination temperature is $32^{\circ} \mathrm{C}$ and the resveratrol content increased with germination time.

UV light is ultraviolet light and is divided into three types: UVA (320-400 nm), UVB (280-320 nm), UVC (200-280 nm) (Surjadinata et al., 2017). UVB is the ultraviolet source that affects organisms at a moderate level and recent studies showed that UVB light induced the biosynthesis of phenolic compounds in mung bean sprouts (Wang et al., 2017), vigna mungo sprouts (Shaukat et al., 2013) and wheat and pea sprouts (Alexieva et al., 2001). To my knowledge, no study has investigated the effect of UVB on bioactive compounds in peanut sprouts. Thus, this study aims to test the hypothesis that UVB exposure induces the accumulation of total phenolics and resveratrol and increase antioxidant activity of peanut sprouts.

\section{Materials and methods}

\subsection{Materials}

Peanut seeds

Three peanut cultivars used for this study were Lac sen, L14 and L27 (grown in Bac Giang and Bac Ninh provinces and harvested in June 2018).

Peanut sprouts

Firstly, peanut seeds were washed three times with water and treated with $70 \%$ ethanol for 30 seconds. The seeds were then washed with water and soaked in a water bath (GFL, Germany) at $30^{\circ} \mathrm{C}$ for six hours. After that, the seeds were germinated in plastic cups (dimension: $9.5 \mathrm{~cm}$ depth $\times 8.5 \mathrm{~cm}$ top diameter x $5.5 \mathrm{~cm}$ base diameter). The seeds were germinated under dark conditions in a box which was placed in a $30^{\circ} \mathrm{C}$-room. The experiment was designed with two formulas: UVB exposure and control (nonUVB exposure). For UVB exposure, sprouts were treated with UVB for one hour every day at 9 am and the UVB lamp was placed in a distance of $20 \mathrm{~cm}$ above the sprouts. UVB lamp was $45 \mathrm{~cm}$ in length, $15 \mathrm{~W}$, 10\% UVB (Repti Glo 10.0, China).

The germinated seeds were collected on days 1, 3 and 5. The experiment was repeated three times. The collected sprouts were then kept at $-22^{\circ} \mathrm{C}$, then freeze-dried for three days by ModulyoD Freeze Dryer (Thermo electron corporation, USA), ground and stored at $-22^{\circ} \mathrm{C}$ for the analysis of phenolic compounds and resveratrol contents and antioxidant activity.

\subsection{Chemicals and reagents}

All chemicals were analytical-graded. 1,1diphenyl-2-picryl-hydrazyl (DPPH) and Trolox were purchased from Sigma (USA). Gallic acid 
and Folin-Ciocalteu reagent were purchased from Merk (Germany). Acetonitrile and methanol were obtained from Samchun (Spain). Other chemicals were from China.

\subsection{Determination of germination rate and sprout length}

The germination rate and the sprout length were measured at harvest time. The germination rate was calculated by the percentage of germinated seeds. The sprout length was measured on 15 randomly selected germinated seeds by a ruler.

\subsection{Determination of total phenolic (TP) content}

Seed and sprout extracts were prepared using the method by Yu et al. (2016) with some modifications. $500 \mathrm{mg}$ of milled seeds or milled sprouts were weighed into a $15-\mathrm{mL}$ Falcon tube and $10 \mathrm{~mL}$ of ethanol $80 \%$ was added. The sample was extracted in 45 minutes at $80^{\circ} \mathrm{C}$ and shaken by using a vortex every 10 minutes. The extract was then centrifuged at $6000 \mathrm{rpm}$ for 20 minutes and the supernatant was collected and stored at $-22^{\circ} \mathrm{C}$.

TP content was measured using the FolinCiocalteu method described by Fu et al. (2011). In brief, $0.5 \mathrm{~mL}$ of the diluted sample was transferred into a test tube and $2.5 \mathrm{~mL}$ of $1: 10$ diluted Folin-Ciocalteu reagent was then added and mixed well. After 4 minutes, $2 \mathrm{~mL}$ of $7.5 \%$ $\mathrm{Na}_{2} \mathrm{CO}_{3}$ was added. The reaction was incubated at room temperature in the dark for $2 \mathrm{~h}$ and the absorbance of the mixture was then measured at $760 \mathrm{~nm}$ using a UV-visible spectrophotometer (UV-Vis 1800, Shimadzu, Japan). A calibration curve was established based on workingstandard solutions of $0.02,0.04,0.06,0.08$ and $0.1 \mathrm{mg} / \mathrm{mL}$ for the calculation of TP content. The results were expressed in gallic acid equivalents per gram dry weight (mg GAE/g DW).

TP content was calculated by the following formulas:

$$
\mathrm{TPC}=\frac{A * V * d F}{a * m} \quad(\text { Eq. } 1)
$$

In which

TPC: Total phenolic content (mg GAE/g DW)

A: Absorbance at $760 \mathrm{~nm}$

$\mathrm{V}$ : Volume of the extract $(\mathrm{mL})$

$\mathrm{dF}$ : Dilution factor

standard curve

a: Slope factor of gallic acid

m: Sample weight (g)

\subsection{Antioxidant capacity measurement}

The extracts of total phenolics were used for antioxidant activity determination. Antioxidant capacity was determined using the 1,1-diphenyl2-picryl-hydrazyl (DPPH) assay described by Thaipong et al. (2006) with some modifications. The stock solution of DPPH was made by dissolving $24 \mathrm{mg}$ of DPPH with $100 \mathrm{~mL}$ of methanol and then kept at $-23^{\circ} \mathrm{C}$ until needed. The working solution of DPPH was prepared by diluting $10 \mathrm{~mL}$ of the stock solution with $45 \mathrm{~mL}$ of methanol. The reaction was carried out by mixing $150 \mu \mathrm{L}$ of diluted sprout extract with $2850 \mu \mathrm{L}$ of the DPPH solution for 30 minutes. A control was also prepared by using $150 \mu \mathrm{L}$ of methanol instead of sprout extract. The absorbance was then measured at $515 \mathrm{~nm}$ using a spectrophotometer (UV-Vis 1800, Shimadzu, Japan). The results were calculated based on a standard curve established from a set of Trolox standard solutions of 50, 100, 250, 500, 750 and $1000 \mu \mathrm{M}$. The results were expressed in Trolox equivalents ( $\mu \mathrm{M}$ TE/g DW).

Antioxidant activity was calculated by the following formulas:

$$
\mathrm{AA}=\frac{A A \% * V * d F}{a * m * 1000} \quad \text { (Eq.2) }
$$

In which

$$
\text { DW) }{ }^{\text {AA: Antioxidant activity }(\mu \mathrm{M} \mathrm{TE} / \mathrm{g}}
$$

AA\%: \% inhibition calculated by this equation

$$
\mathrm{AA} \%=\frac{(\text { Acontrol }- \text { Asample }) * 100}{\text { Acontrol }}(\mathrm{Eq} .3)
$$


$\mathrm{V}$ : Volume of the extract $(\mathrm{mL})$

dF: Dilution factor

a: Slope factor of Trolox standard curve equation

m: Sample weight (g)

1000: Conversion factor from $\mathrm{mL}$ to $\mathrm{L}$ (of the extract).

\subsection{Determination of resveratrol content}

Samples were extracted as described for the total phenolic extraction. Resveratrol content was determined using the Agilent 1260 Series HPLC system equipped with a UV detector (Agilent Technologies) and LC - Solution software. The column used was Kinetex $5 \mu \mathrm{m}$ EVO C18 $100 \AA, 150 \times 4.6 \mu \mathrm{M}$. The wavelength and column temperature were set at $306 \mathrm{~nm}$ and $30^{\circ} \mathrm{C}$, respectively. The injection volume and the flow rate were $40 \mu \mathrm{L}$ and $1 \mathrm{~mL} /$ minute, respectively. The mobile phase was a gradient (as described in Table 1) of deionized water mixed with formic acid (0.1\%) (phase A) and acetonitrile mixed with formic acid $(0.1 \%)$ (phase B). The results were calculated based on a standard curve established from a set of resveratrol standard solutions: $0.76,1.56,3.125$, 6.25 and $12.5 \mu \mathrm{g} / \mathrm{mL}$ and their peak areas. The results were expressed in $\mu \mathrm{g} / \mathrm{g}$ DW.

Resveratrol content was calculated as the following equation:

$$
\text { Resveratrol content }=\frac{S * V * d F}{a * m}(\text { Eq.4) }
$$

In which

Resveratrol content ( $\mu \mathrm{g} / \mathrm{g}$ DW)

S: Peak area

$\mathrm{V}$ : Volume of the extract $(\mathrm{mL})$

$\mathrm{dF}$ : Dilution factor standard curve

a: Slope factor of resveratrol m: Sample weight (g)
Table 1. The gradient of mobile phase

\begin{tabular}{|l|l|}
\hline Time (minutes) & Phase B (\%) \\
\hline 0 & 0 \\
\hline 5 & 15 \\
\hline 20 & 20 \\
\hline 30 & 100 \\
\hline 35 & 100 \\
\hline 40 & 0 \\
\hline 42 & 0 \\
\hline 42.01 & 0 \\
\hline
\end{tabular}

\subsection{Statistical analysis}

Data and comparisons were analyzed using $\mathrm{R}$ version 3.5.0. Sprout variables were analysed by three-way independent ANOVA (Cultivar $\mathrm{x}$ Germination time $x$ UVB). Significant differences between the means were analysed by Duncan's test $(\mathrm{P}<0.05)$. The particular set of variables (TP content and 100-seed weight) was subjected to Pearson's correlation analysis (Field, 2013).

\section{Results and discussions}

\subsection{Hundred-seed weight, TP and resveratrol contents and antioxidant activities of seeds}

Significant $(\mathrm{P}<0.05)$ variations in the TP content and antioxidant activity of seeds were observed between the three peanut cultivars (Table 2). Lac sen seeds had the highest TP content ( $4.73 \pm 0.19 \mathrm{mg} \mathrm{GAE} / \mathrm{g} \mathrm{DW})$, while L14 seeds had the lowest TP content $(2.97 \pm 0.20 \mathrm{mg}$ GAE/g DW). The trend of antioxidant activity was different from that of TP content in the three cultivars, in which Lac sen seeds had the highest antioxidant activity $(3.46 \pm 0.07 \mu \mathrm{M} \mathrm{TE} / \mathrm{g} \mathrm{DW})$, and the antioxidant activity was the lowest for L27 seed $(2.40 \pm 0.17 \mu \mathrm{M}$ TE/g DW $)$. The higher seed TP content might be due to the lower 100-seed weight (Table 2) because the TP content of seeds had significantly negative correlation with 100 -seed weight $\left(r^{2}=-0.92\right.$, 
$\mathrm{P}<0.001)$. Phenolic compounds mainly exist in peanut skins (Khaopha et al., 2012) and smaller seeds have more skins than larger seeds; thus, smaller seeds result in higher TP contents than larger seeds.

Table 2. Hundred-seed weight, TP and resveratrol content and antioxidant activity of seeds of three peanut cultivars. Data represent means \pm standard deviations $(n=3$, except $n=1$ for resveratrol content).

\begin{tabular}{|l|l|l|l|l|}
\hline Cultivar & $\begin{array}{l}100 \text {-seed weight } \\
(\mathrm{g})\end{array}$ & $\begin{array}{l}\text { TP content }(\mathrm{mg} \\
\text { GAE/g DW })\end{array}$ & $\begin{array}{l}\text { Resveratrol content } \\
(\mu \mathrm{g} / \mathrm{g} \mathrm{DW})\end{array}$ & $\begin{array}{l}\text { Antioxidant activity } \\
(\mu \mathrm{M} \text { TE/g DW })\end{array}$ \\
\hline Lac sen & $46.83 \pm 0.76^{\mathrm{b}}$ & $4.73 \pm 0.19^{\mathrm{a}}$ & 4.20 & $3.46 \pm 0.07^{\mathrm{a}}$ \\
\hline L14 & $65.30 \pm 5.79^{\mathrm{a}}$ & $2.97 \pm 0.20^{\mathrm{b}}$ & 7.41 & $2.89 \pm 0.13^{\mathrm{b}}$ \\
\hline L27 & $59.65 \pm 1.13^{\mathrm{a}}$ & $3.89 \pm 0.16^{\mathrm{c}}$ & 5.92 & $2.40 \pm 0.17^{\mathrm{c}}$ \\
\hline
\end{tabular}

Different letters show significant differences within each column $(P<0.05)$.

Previous studies showed TP contents ranged from about 0.10-2.30 mg GAE/g fresh weight among 20 peanut cultivars (Chukwumah et al., 2009), 0.15-3.04 mg GAE/g DW among 15 genotypes (Khaopha et al., 2012) and 0.15-0.53 mg GAE/g DW among 5 genotypes (Adhikari et al., 2018). Khaopha et al. (2012) reported that TP contents mainly present in peanut skins and the color of peanut skin had strong correlation with the TP content of peanut kernels (Chukwumah et al., 2009) and peanut skins with pink color contained significantly higher TP contents than those with gray and yellow Khaopha et al. (2012). Peanut cultivars in our study had pink color and had slightly higher TP contents $(2.97 \pm 0.20-4.73 \pm 0.19 \mathrm{mg} \mathrm{GAE} / \mathrm{g}$ DW) compared to those with pink color (1.83-
$3.04 \mathrm{mg} \mathrm{GAE} / \mathrm{g}$ DW) reported by Khaopha et al. (2012). Similar to our study, Craft et al. (2010) showed antioxidant activities also varied (3.02$11.9 \mu \mathrm{M}$ TE/g DW) among 8 peanut cultivars.

Seed resveratrol contents varied (0.13-3.4 $\mu \mathrm{g} / \mathrm{g}$ DW) depending on cultivars (Adhikari et al., 2018; Yu et al., 2016; Wang and Pittman, 2009). In this study, the resveratrol content of seeds ranged from 4.20 to $7.41 \mu \mathrm{g} / \mathrm{g} \mathrm{DW}$, in which Lac sen seeds had the lowest resveratrol content, whereas that of L14 was the highest (Table 2). In our study, the peanuts cultivars had higher resveratrol contents compared to previous reports (Adhikari et al., 2018; Yu et al., 2016; Wang and Pittman, 2009). This might be attributed to genotypes or agronomic conditions.

\subsection{Germination rate and sprout length}

Table 3. Effect of cultivar, germination time and UVB on germination rate, sprout length, TP and resveratrol contents and antioxidant activity. Germination rate is measured in percentage; sprout length is measured in $\mathrm{cm}$; TP content is measured in $\mathrm{mg} \mathrm{GAE} / \mathrm{g} \mathrm{DW}$; resveratrol content is measured in $\mu \mathrm{g}$ resveratrol/g DW; antioxidant activity is measured in $\mu \mathrm{M} \mathrm{TE} / \mathrm{g} \mathrm{DW} .{ }^{*}, * *$ and $* * *$ significant at $\mathrm{P}<0.05, \mathrm{P}<0.01$ and $\mathrm{P}<0.001$, respectively.

\begin{tabular}{|c|c|c|c|c|c|}
\hline & $\begin{array}{l}\text { Germination } \\
\text { rate }\end{array}$ & $\begin{array}{l}\text { Sprout } \\
\text { length }\end{array}$ & TP content & $\begin{array}{l}\text { Resveratrol } \\
\text { content }\end{array}$ & $\begin{array}{l}\text { Antioxidant } \\
\text { activity }\end{array}$ \\
\hline \multicolumn{6}{|c|}{ Cultivar (C) } \\
\hline Lac sen & 88.9 & 4.88 & 4.02 & 40.44 & 1.00 \\
\hline
\end{tabular}


Nguyen et al./ Carpathian Journal of Food Science and Technology, 2020, 12(1), 89-101

\begin{tabular}{|c|c|c|c|c|c|}
\hline L14 & 83.9 & 5.28 & 3.81 & 25.84 & 1.29 \\
\hline L27 & 95.2 & 5.52 & 3.51 & 26.30 & 1.35 \\
\hline \multicolumn{6}{|l|}{ Germination time (GT) } \\
\hline D1 & 83.1 & 1.82 & 3.12 & 10.17 & 0.86 \\
\hline D3 & 93.5 & 4.59 & 3.77 & 20.50 & 1.25 \\
\hline D5 & 91.2 & 9.26 & 4.44 & 61.91 & 1.53 \\
\hline \multicolumn{6}{|l|}{$U V B$} \\
\hline Control & 89.4 & 5.4 & 3.6 & 23.34 & 1.07 \\
\hline UVB exposure & 89.3 & 5.1 & 4.0 & 38.37 & 1.35 \\
\hline \multicolumn{6}{|l|}{ F ratio } \\
\hline Cultivar (C) & $11.30^{* * * *}$ & $4.58^{*}$ & $11.95^{* * *}$ & $27.70^{* * *}$ & $42.47^{* * *}$ \\
\hline Germination time (GT) & $10.52^{* * *}$ & $620.35^{* *}$ & $77.67^{* * *}$ & $93.37^{* * *}$ & $462.18^{* * *}$ \\
\hline UVB & 0.00 & 3.27 & $16.22^{* * *}$ & $46.50^{* * *}$ & $104.49^{* * *}$ \\
\hline $\mathrm{C} \times \mathrm{GT}$ & $9.21^{* * *}$ & $5.24^{* *}$ & $3.95^{* *}$ & $6.52^{* * *}$ & $25.54^{* * *}$ \\
\hline $\mathrm{C} \times \mathrm{UVB}$ & 1.24 & 1.35 & 0.50 & 0.31 & $5.95^{* *}$ \\
\hline $\mathrm{GT} \times \mathrm{UVB}$ & 0.31 & 2.60 & 0.05 & 2.43 & $34.15^{* * * *}$ \\
\hline $\mathrm{C} \times \mathrm{GT} \times \mathrm{UVB}$ & 1.40 & 0.36 & 1.14 & 0.24 & $3.44^{*}$ \\
\hline
\end{tabular}

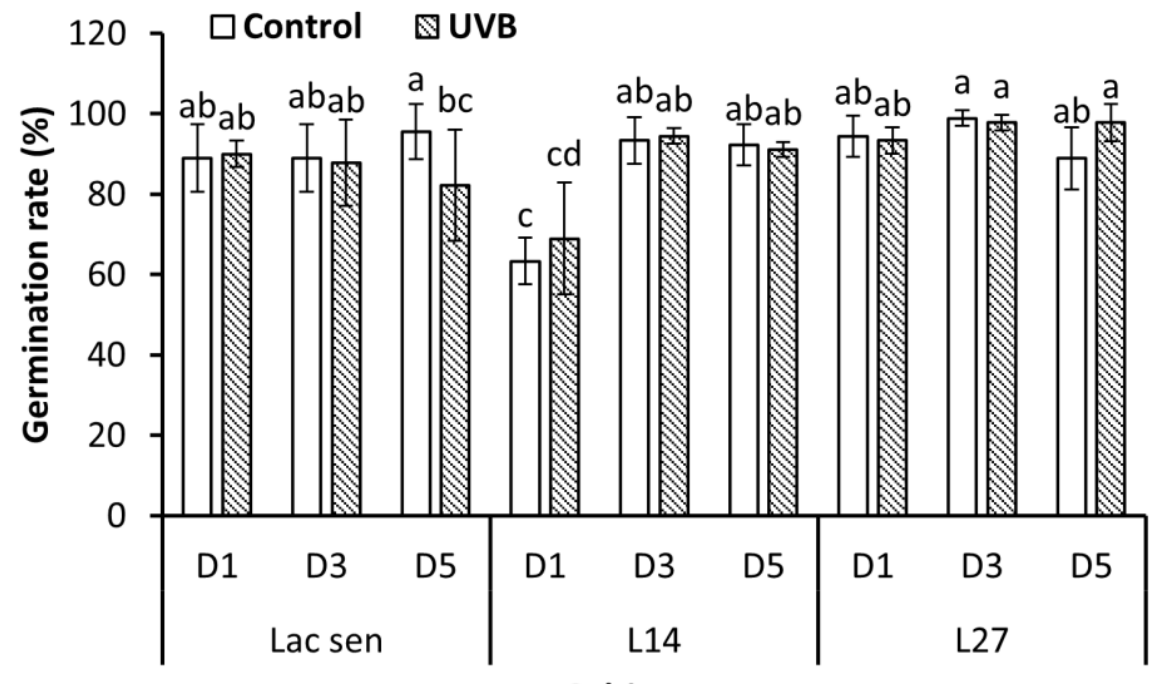

Cultivar

Figure 1. Effect of germination time and UVB exposure on germination rates of three peanut cultivars. Data represent means \pm standard deviations $(n=3)$. Different letters show significant differences $(P<0.05)$. 


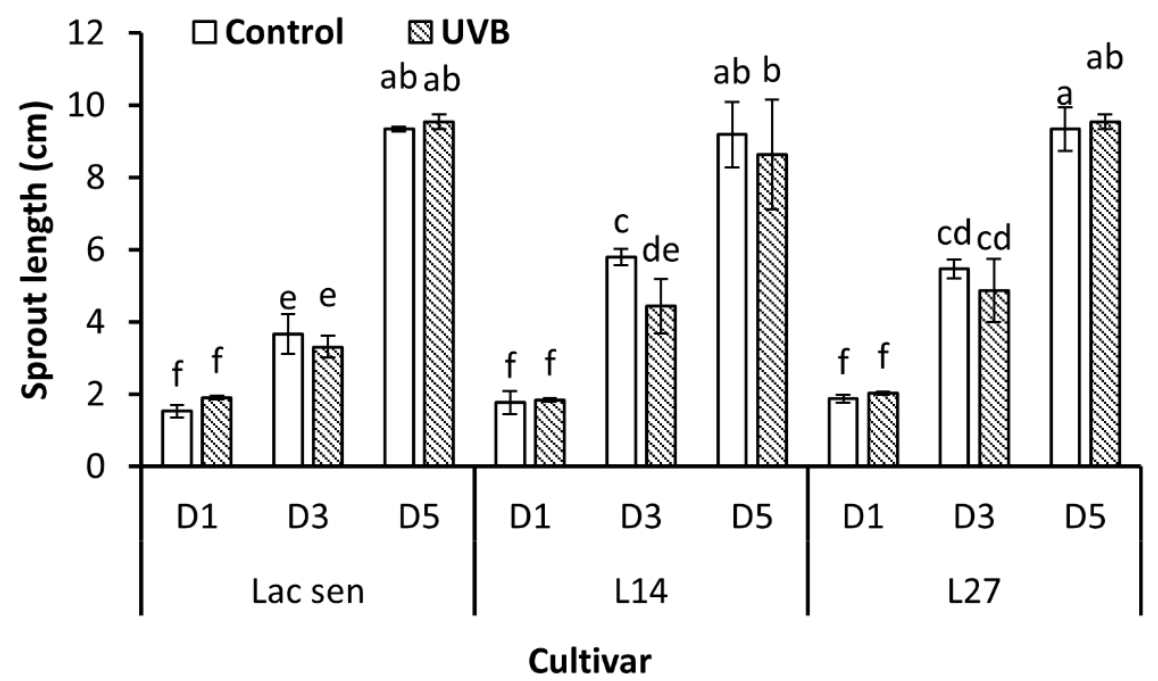

Figure 2. Effect of germination time and UVB exposure on sprout length of three peanut cultivars. Data represent means \pm standard deviations $(n=3)$. Different letters show significant differences $(P<0.05)$.

UVB exposure had no effects on germination rate and sprout length, while the cultivar (C) and germination time (GT) significantly affected these two parameters (Table 3). Significant interaction between cultivar and germination time was observed. This means cultivars behave differently in germination rate and sprout length during germination. Indeed, although the germination rates of two cultivars Lac sen and L27 were not different between germination periods, averagely about $90 \%$, this variable of L14 was significantly lower at day 1 (D1) (about 65\%) compared to day 3 (D3) and day 5 (D5) (Figure 1). Sprout length increased dramatically during kernel germination, ranging from $2 \mathrm{~cm}$ at D1 to about $9 \mathrm{~cm}$ at D5.

There were no significant differences in sprout length between cultivars at D1 and D5, but at D3, this variable of Lac sen was lower than that of the other two cultivars (Figure 2).

\subsection{TP and resveratrol contents and antioxidant activities}

All three factors: cultivar, germination time and UVB exposure significantly affected the TP content, the resveratrol content and the antioxidant activity (Table 2). No significant $\mathrm{C}$ $x$ UVB, GT $x$ UVB and $C \times$ GT $x$ UVB interactions occurred for $\mathrm{TP}$ content and resveratrol content, but $\mathrm{C}$ x GT interactions were found for these two variables. Significant $C x$ GT, C x UVB, GT x UVB and C x GT x UVB interactions were observed for antioxidant activity.

TP contents of all cultivars significantly increased through D1 to D5 (Table 3, Table 4). On average, the TP content enhanced $42.31 \%$ at D5 compared to D1 (Table 3), but the changes varied between cultivars and by UVB treatments. Under control conditions, the TP content of L27 increased by $27.3 \%$ from D1 (3.00 $\mathrm{mg} \mathrm{GAE} / \mathrm{g} \mathrm{DW}$ ) to D5, while these increases were higher with $54.8 \%$ and $57.7 \%$ for Lac sen and L14, respectively. Under UVB exposure, an increase in TP content from D1 $(3.60 \pm 0.48 \mathrm{mg} \mathrm{GAE} / \mathrm{g} \mathrm{DW})$ to D5 was $35.3 \%$ for Lac sen, lower than the increases in L27 and L14 with $40.6 \%$ and $41.6 \%$, respectively. Increases in TP content in 13 different germinated seeds have been reported (CevallosCasals and Cisneros-Zevallos, 2010). However, Limmongkon et al. (2017) indicated that phenolic accumulation of five peanut cultivars 
during germination did not show any consistent trend through D1 to D4. For example, TP content of Tainan increased from D1 to D4, while that of Kalasin1 decreased from D1 to D3 then increased. The TP contents of sprout cotyledon and sprout without cotyledon also varied among six peanut cultivars through D5 to D9 (Adhikari et al., 2018).

Abiotic stresses including UVB exposure stimulate the biosynthesis of phenolic compounds (Surjadinata et al., 2017). In our study, UVB exposure led to significantly increasing the TP content by $11.11 \%$ (Table 3 ). Cultivars did not show consistent trend in TP contents through D1 to D5 (Table 4). For Lac sen, UVB exposure resulted in significant increases in TP contents of $18.0 \%$ and $16.8 \%$ at D1 and D3, respectively, but showed no effect at D5. However, UVB exposure had no significant impact on TP contents of L14 sprouts at any days. UVB exposure did not affect TP contents of L27 sprouts at D1 and D3, but significantly led to a rise of $17.0 \%$ at D5. The accumulation of phenolics is a protective mechanism of plants to respond to UVB exposure (Escobar-Bravo et al., 2017). UVB exposure also led to enhancements of TP contents in mung bean sprouts (Wang et al., 2017), vigna mungo sprouts (Shaukat et al., 2013) and wheat and pea sprouts (Alexieva et al., 2001). Previous studies found UVB exposure resulted in the increases of kaempferol (2.1-folds) and quercetin (1.5-folds) (flavonoids) in broccoli sprouts (Mewis et al., 2012), and an increase of anthocyanins, about 3folds and 2-folds in wheat and pea sprouts, respectively (Alexieva et al., 2001). Therefore, UVB exposure could be a potential approach to increase beneficial phenolics in seed sprouts.

Table 4. Effect of germination time and UVB exposure on TP content of three peanut cultivars. Data represent means \pm standard deviations $(n=3)$. Different capital letters show significant differences within each column $(P<0.05)$; different small letters show significant differences in each row $(P<0.05)$.

\begin{tabular}{|l|l|l|l|l|}
\hline \multirow{2}{*}{ Cultivar } & \multirow{2}{*}{$\begin{array}{c}\text { UVB } \\
\text { exposure }\end{array}$} & \multicolumn{3}{|c|}{ TP content $(\mathrm{mg}$ GAE/g DW) } \\
\cline { 3 - 5 } & & $\mathrm{D} 1$ & $\mathrm{D} 3$ & $\mathrm{D} 5$ \\
\hline Lac sen & UVB & $3.60 \pm 0.48^{\mathrm{Ac}}$ & $4.25 \pm 0.10^{\mathrm{Ab}}$ & $4.87 \pm 0.05^{\mathrm{Aa}}$ \\
\hline & Control & $3.05 \pm 0.12^{\mathrm{Bc}}$ & $3.64 \pm 0.24^{\mathrm{BCDb}}$ & $4.72 \pm 0.34^{\mathrm{Aa}}$ \\
\hline L14 & UVB & $3.15 \pm 0.16^{\mathrm{ABb}}$ & $4.14 \pm 0.33^{\mathrm{ABa}}$ & $4.46 \pm 0.33^{\mathrm{ABa}}$ \\
\hline & Control & $2.72 \pm 0.12^{\mathrm{Bb}}$ & $4.04 \pm 0.07^{\mathrm{ABCa}}$ & $4.29 \pm 0.63^{\mathrm{ABa}}$ \\
\hline L27 & UVB & $3.18 \pm 0.25^{\mathrm{ABb}}$ & $3.42 \pm 0.15^{\mathrm{CDb}}$ & $4.47 \pm 0.26^{\mathrm{Aa}}$ \\
\hline & Control & $3.00 \pm 0.21^{\mathrm{Bb}}$ & $3.13 \pm 0.65^{\mathrm{Dab}}$ & $3.82 \pm 0.34^{\mathrm{Ba}}$ \\
\hline
\end{tabular}

Resveratrol is a phenolic naturally found in peanut seeds, but its content is low (Sales and Resurreccion, 2014). Studies revealed the content of this compound can be enhanced by seed germination (Hasan and Bae, 2017; Wang et al., 2005). Our study found that the resveratrol content increased $508.75 \%$ at D5 compared to that at D1 (Table 3). The increases varied between cultivars and by UVB treatments
(Table 5). Under both UVB exposure and control conditions, resveratrol contents of all three cultivars increased dramatically from D1 through D5. Under nonUVB exposure, resveratrol content of Lac sen sprouts at D5 had an enhancement of 6.2-fold compared to that of D1 $(17.59 \mu \mathrm{g} / \mathrm{g}$ DW). L27 sprouts also showed a 7.0-fold increase in resveratrol content at D5 compared to D1 $(5.57 \mu \mathrm{g} / \mathrm{g}$ DW), while the increase in this variable for L14 sprouts at D5 
compared to D1 (8.37 $\mu \mathrm{g} / \mathrm{g} \mathrm{DW})$ was 4.4-fold. Under UVB exposure, resveratrol contents also increased 5.9-, 6.9- and 6.1-fold at D5 compared to D1 for Lac sen, L14 and L27 sprouts, respectively; resveratrol contents of these cultivars at D1 were $17.59,9.50$ and $9.57 \mu \mathrm{g} / \mathrm{g}$ DW respectively. The enhancements of resveratrol content in our study are similar to findings in three peanut cultivars in the study by Wang et al. (2005), in which cultivar Tainan 11 showed an increase from $3.7 \mu \mathrm{g} / \mathrm{g}$ DW at D0 to $17.7 \mu \mathrm{g} / \mathrm{g}$ DW at D6. Another research reported peanut sprouts at D5 reached resveratrol content of $32.87 \mu \mathrm{g} / \mathrm{g}$ DW, which was about 9.8 -fold higher than that in non-germinated peanuts ( $\mathrm{Yu}$ et al., 2016). Similarly, resveratrol contents in sprouts of six peanut genotypes at D5 were higher than that in seeds (Adhikari et al., 2018). However, Limmongkon et al. (2017) found two of five peanut cultivars had downward trends in resveratrol content from D1 to D4 of germination, while the other three cultivars did not show any consistent trend. These differences could be due to differences in genotypes.

Resveratrol is synthesized in plants in response to abiotic stresses (Hasan and Bae, 2017), but to our knowledge, no study has focused on the effect of UVB light on resveratrol response in peanut sprouts. Our study showed that UVB exposure significantly enhanced the accumulation of resveratrol by $62.81 \%$ on average (Table 3 ). The enhancements differed at different germination time and between cultivars. At D1, UVB exposure resulted in significant increases in resveratrol contents in Lac sen and L27, but not in L14. The increases by UVB treatment occurred in all cultivars at D3 and D5. At D5, UVB exposure led to the highest increase of resveratrol content with $78.2 \%$ in Lac sen, followed by L14 with $77.0 \%$ and L27 with $49.2 \%$. The accumulation of resveratrol in peanut seedlings and peanut leaves increased 196-fold and 200-fold, respectively in response to UV light (Tang et al., 2010; Chung et al., 2003). Another study showed UV exposure on sliced peanuts induced the synthesis of resveratrol (Potrebko and Resurreccion, 2009). Post-harvest treatment with UVB enhanced resveratrol in grapes two-fold (Cantos et al., 2000). According to Tang et al. (2010), resveratrol is considered to be associated with plant defense responses because it is induced by to stresses. Accumulation of resveratrol could be to a factor to alleviate reactive oxygen species (ROS) induced by UVB exposure, thus preventing plant tissues from damages.

Table 5. Effect of germination time and UVB exposure on resveratrol content of three peanut cultivars. Data represent means \pm standard deviations $(n=3)$. Different capital letters show significant differences within each column $(P<0.05)$; different small letters show significant differences in each row

$(P<0.05)$.

\begin{tabular}{|l|l|l|l|l|}
\hline \multirow{2}{*}{ Cultivar } & \multirow{2}{*}{$\begin{array}{c}\text { UVB } \\
\text { exposure }\end{array}$} & \multicolumn{3}{|c|}{ Resveratrol content $(\mu \mathrm{g}$ resveratrol/g DW) } \\
\cline { 3 - 5 } & & $\mathrm{D} 1$ & $\mathrm{D} 3$ & $\mathrm{D} 5$ \\
\hline Lac sen & UVB & $17.59 \pm 2.70^{\mathrm{Ab}}$ & $26.84 \pm 2.84^{\mathrm{Ab}}$ & $110.16 \pm 18.6^{\mathrm{Aa}}$ \\
\hline & Control & $10.43 \pm 1.33^{\mathrm{Bb}}$ & $15.76 \pm 0.90^{\mathrm{Bb}}$ & $61.82 \pm 6.54^{\mathrm{Ba}}$ \\
\hline L14 & UVB & $9.50 \pm 0.86^{\mathrm{Bc}}$ & $19.51 \pm 3.05^{\mathrm{Bb}}$ & $65.24 \pm 4.25^{\mathrm{Ba}}$ \\
\hline & Control & $8.37 \pm 0.61^{\mathrm{Bc}}$ & $15.52 \pm 0.14^{\mathrm{Bb}}$ & $36.85 \pm 2.16^{\mathrm{Ca}}$ \\
\hline L27 & UVB & $9.57 \pm 0.32^{\mathrm{Bc}}$ & $28.62 \pm 3.12^{\mathrm{Ab}}$ & $58.29 \pm 6.32^{\mathrm{Ba}}$ \\
\hline & Control & $5.57 \pm 0.44^{\mathrm{Cc}}$ & $16.69 \pm 2.40^{\mathrm{Bb}}$ & $39.06 \pm 5.47^{\mathrm{Ca}}$ \\
\hline
\end{tabular}


Antioxidants are considered to be radical scavenger which reacts readily with radicals or ROS and the present of these substances is usually measured by antioxidant activity (Sindhi et al., 2013). Accumulation of antioxidants is a protective mechanism of plants in response to stresses (Das and Roychoudhury, 2014). In our current study, UVB exposure was found to increase antioxidant activity by $26.17 \%$ on average (Table 3); however, the effect of UVB was different among cultivars (Table 6). UVB exposure led to a significant increase of antioxidant activity in Lac sen sprouts at D3 from $0.92 \pm 0.11$ to $1.32 \pm 0.22 \mu \mathrm{M}$ TE/g DW, while no significant enhancement occurred at D1 and D5. UVB treatment increased antioxidant activity of L14 sprouts by $34.6 \%$ at D1, whereas this variable was not significantly affected at D3 and D5. An enhancement of $18.7 \%$ was observed in L27 sprouts at D5 by UVB exposure, but this did not occur at D1 and D3. To our knowledge, no information has been reported on the effect of UVB exposure on the antioxidant activity in seed sprouts, but previous studies found the antioxidant activity of tobacco leaves enhanced after the plants were treated with UVB (Shen et al., 2017) and the antioxidant activity in the plants, Deschampsia antarctica Desv. enhanced after $3 \mathrm{~h}$ of exposure to UVB (Köhler et al., 2017). Post-harvest UVB exposure also increased antioxidant activities of whole, sliced and peeled carrots (AvenaBustillos et al., 2012; Surjadinata et al., 2017). The enhancements of antioxidant activity by UVB treatments in the peanuts sprouts as well as in the plant tissues might be associated with the increases of antioxidants, such as phenolics. Enhanced antioxidant activities could also be a way of plants to alleviate ROS when exposed to UVB.

Similar to $\mathrm{TP}$ and resveratrol contents, antioxidant activities also increased during the germination of peanut cultivars; this variable enhanced at D5 by $77.91 \%$ compared to D1 (Table 3). Lac sen showed greater increase in antioxidant activities at D5 than the other cultivars under both treatment conditions (Table 6 ). The values of this variable in Lac sen sprouts significantly increased by $207.1 \%$ and $166.1 \%$ at D5 compared to D1 under control conditions and UVB exposure, respectively; followed by L14 sprouts with $83.3 \%$ and $56.2 \%$, while the enhancements in L27 sprouts were the lowest with $40.2 \%$ and $44.7 \%$. The results from this study are in agreement with a report by Wang et al. (2005) where antioxidant activities of three peanut cultivars enhanced with an increase of germination time. In another study, antioxidant activities of all seven peanut cultivars at D10 of germination were significantly higher than those at D0 (soaked peanuts) (Yang et al., 2019). A study on 13 other seeds also showed significantly increased antioxidant activities in all sprouts after seven days of germination compared to soaked and original seeds (Cevallos-Casals and Cisneros-Zevallos, 2010). The increase of antioxidant activity could be related to the enhancement of antioxidants such as phenolic compounds, and therefore peanut sprouts can be potential sources for functional food production.

Table 6. Effect of germination time and UVB exposure on resveratrol content of three peanut cultivars. Data represent means \pm standard deviations $(n=3)$. Different capital letters show significant differences within each column $(P<0.05)$; different small letters show significant differences in each row

$(P<0.05)$.

\begin{tabular}{|l|l|l|l|l|}
\hline \multirow{2}{*}{ Cultivar } & \multirow{2}{*}{$\begin{array}{l}\text { UVB } \\
\text { exposure }\end{array}$} & \multicolumn{4}{|l|}{ Antioxidant activity $(\mu \mathrm{M}$ TE/g DW) } \\
\cline { 3 - 5 } & D1 & D3 & D5 \\
\hline Lac sen & UVB & $0.56 \pm 0.06^{\mathrm{Db}}$ & $1.32 \pm 0.22^{\mathrm{ABa}}$ & $1.49 \pm 0.22^{\mathrm{BCa}}$ \\
\hline
\end{tabular}




\begin{tabular}{|c|c|c|c|c|}
\hline & Control & $0.42 \pm 0.15^{\mathrm{Dc}}$ & $0.92 \pm 0.11^{\mathrm{Cb}}$ & $1.29 \pm 0.07^{\mathrm{Ca}}$ \\
\hline \multirow[t]{2}{*}{ L14 } & UVB & $1.05 \pm 0.05^{\mathrm{Bb}}$ & $1.63 \pm 0.22^{\mathrm{BCa}}$ & $1.64 \pm 0.06^{\mathrm{ABa}}$ \\
\hline & Control & $0.78 \pm 0.15^{\mathrm{Cb}}$ & $1.16 \pm 0.18^{\mathrm{Ba}}$ & $1.43 \pm 0.24^{\mathrm{BCa}}$ \\
\hline \multirow[t]{2}{*}{ L27 } & UVB & $1.23 \pm 0.06^{\mathrm{Ab}}$ & $1.40 \pm 0.23^{\mathrm{ABb}}$ & $1.78 \pm 0.11^{\mathrm{Aa}}$ \\
\hline & Control & $1.07 \pm 0.05^{\mathrm{ABb}}$ & $1.07 \pm 0.10^{\mathrm{BCb}}$ & $1.50 \quad 0.07^{\mathrm{BCa}}$ \\
\hline
\end{tabular}

\section{Conclusions}

This study revealed both germination time and UVB exposure increased TP and resveratrol contents and antioxidant activities. TP and resveratrol contents and the antioxidant activity at D5 increased $42.31 \%, 508.75 \%$ and $77.91 \%$ on average compared to D1, respectively, while UVB exposure enhanced TP and resveratrol contents and the antioxidant activity by $11.11 \%$, $62.81 \%$ and $26.17 \%$ on average. Peanut cultivars responded differently to germination time and UVB exposure. UVB exposure significantly enhanced TP content of L27 sprouts at D5 by $17.0 \%$ but this effect did not occur in the other cultivars. UVB exposure significantly induced the accumulation of resveratrol in D5 sprouts of all cultivars, in which Lac sen sprouts at D5 showed the highest content with $110.16 \mu \mathrm{g} / \mathrm{g}$ DW. UVB exposure also significantly enhanced the antioxidant activity of L27 at D5, but the effect was not found in the other two cultivars. UVB exposure induced the accumulation of phenolics, particularly resveratrol in peanut sprouts, suggesting that peanut sprouts can be potential sources for the production functional foods.

\section{References}

Adhikari, B., Dhungana, S.K., Ali, M.W., et al. (2018) Resveratrol, total phenolic and flavonoid contents, and antioxidant potential of seeds and sprouts of Korean peanuts. Food Science and Biotechnology.

Aggarwal, B.B., Bhardwaj, A., Aggarwal, R.S., et al. (2004) Role of resveratrol in prevention and therapy of cancer, Preclinical and clinical studies. Anticancer Research 24, 2783-2840.
Alexieva, V., Sergiev, I., Mapelli, S., et al. (2001) The effect of drought and ultraviolet radiation on growth and stress markers in pea and wheat. Plant Cell and Environment 24, 1337-1344.

Avena-Bustillos, RJ, Du WX, Woods R, et al. (2012) Ultraviolet-B light treatment increases antioxidant capacity of carrot products. Journal of the Science of Food Agriculture 92, 2341-2348.

Cantos, E., Garcia-Viguera, C., de PascualTeresa, S., et al. (2000) Effect of postharvest ultraviolet irradiation on resveratrol and other phenolics of cv. Napoleon table grapes. Journal of Agriculture and Food Chemistry 48, 4606-4612.

Cevallos-Casals, B.A. and Cisneros-Zevallos, L. (2010) Impact of germination on phenolic content and antioxidant activity of 13 edible seed species. Food Chemistry 119, 14851490.

Chukwumah, Y., Walker, L.T. and Verghese, M. (2009) Peanut skin color, a biomarker for total polyphenolic content and antioxidative capacities of peanut cultivars. International Journal of Molecular Sciences 10, 49414952.

Chung, I-M., Park, M.R., Chun, J.C., et al. (2003) Resveratrol accumulation and resveratrol synthase gene expression in response to abiotic stresses and hormones in peanut plants. Plant Science 164, 103-109.

Craft, B.D., Kosinska, A., Amarowicz, R., et al. (2010) Antioxidant properties of extracts obtained from raw, dry-roasted, and oilroasted US peanuts of commercial 
importance. Plant Foods for Human Nutrition 65, 311-318.

Das, K. and Roychoudhury, A. (2014) Reactive oxygen species (ROS) and response of antioxidants as ROS-scavengers during environmental stress in plants. Frontiers in Environmental Science 2.

Escobar-Bravo, R., Klinkhamer, P.G.L. and Leiss, K.A. (2017) Interactive Effects of UV-B Light with Abiotic Factors on Plant Growth and Chemistry, and Their Consequences for Defense against Arthropod Herbivores. Frontiers in Plant Science 8.

Field, A. (2013) Discovering Statistics using IBM SPSS Statistics, Sage Publications Ltd.

$\mathrm{Fu}$, L., Xu，B-T., Xu，X-R., et al. (2011) Antioxidant capacities and total phenolic contents of 62 fruits. Food Chemistry 129, 345-350.

Ha, A.W., Kim, W.K., Kim, J.H., et al. (2015) The supplementation effects of peanut sprout on reduction of abdominal fat and health indices in overweight and obese women. Nutrition Research and Practice 9, 249-255.

Hasan, M. and Bae, H. (2017) An Overview of Stress-Induced Resveratrol Synthesis in Grapes, Perspectives for ResveratrolEnriched Grape Products. Molecules 22.

Hasan, M.M., Cha M, Bajpai VK, et al. (2013) Production of a major stilbene phytoalexin, resveratrol in peanut (Arachis hypogaea) and peanut products, a mini review. Reviews in Environmental Science and Bio-Technology 12, 209-221.

Khaopha, S., Senawong, T., Jogloy, S., et al. (2012) Comparison of total phenolic content and composition of individual phenolic acids in testae and testa-removed kernels of 15 Valencia-type peanut (Arachis hypogaea L.) genotypes. African Journal of Biotechnology 11, 15923-15930.

King, R.E., Bomser, J.A. and Min, D.B. (2006) Bioactivity of resveratrol. Comprehensive Reviews in Food Science and Food Safety 5 , 65-70.
Köhler, H., Contreras, R.A., Pizarro, M., et al. (2017) Antioxidant Responses Induced by UVB Radiation in Deschampsia antarctica Desv. Frontiers in Plant Science 8.

Krishna, G., Singh, B.K., Kim, E.K., et al. (2015) Progress in genetic engineering of peanut (Arachis hypogaea L.)-A review. Plant Biotechnology Journal 13, 147-162.

Limmongkon, A., Janhom, P., Amthong, A., et al. (2017) Antioxidant activity, total phenolic, and resveratrol content in five cultivars of peanut sprouts. Asian Pacific Journal of Tropical Biomedicine 7, 332-338.

Lin, P.Y. and Lai, H.M. (2006) Bioactive compounds in legumes and their germinated products. Journal of Agricultural and Food Chemistry 54, 3807-3814.

Mewis, I., Schreiner, M., Nguyen, C.N., et al. (2012) UV-B irradiation changes specifically the secondary metabolite profile in broccoli sprouts, induced signaling overlaps with defense response to biotic stressors. Plant \& cell physiology 53, 15461560.

Pennington, J.A.T. (2002) Food composition databases for bioactive food components. Journal of Food Composition and Analysis $15,419-434$.

Potrebko, I. and Resurreccion, A.V. (2009) Effect of ultraviolet doses in combined ultraviolet-ultrasound treatments on transresveratrol and trans-piceid contents in sliced peanut kernels. J Agric Food Chem 57, 7750-7756.

Sales, J.M. and Resurreccion, A.V.A. (2014) Resveratrol in Peanuts. Critical Reviews in Food Science and Nutrition 54, 734-770.

Shaukat, S.S., Farooq, M.A., Siddiqui, M.F., et al. (2013) Effect of enhanced UVB radiation on germination, seedling growth and biochemical responses of Vigna mungo (L.) Hepper. Pakistan Journal of Botany 45, 779785.

Shen, J., Jiang, C.Q., Yan, Y.F., et al. (2017) Effect of increased UV-B radiation on carotenoid accumulation and total 
antioxidant capacity in tobacco (Nicotiana tabacum L.) leaves. Genetics and Molecular Research 16.

Sindhi, V., Gupta, V., Sharma, K., et al. (2013) Potential applications of antioxidants - A review. Journal of Pharmacy Research 7, 828-835.

Surjadinata, B.B., Jacobo-Velázquez, D.A. and Cisneros-Zevallos, L. (2017) UVA, UVB and UVC Light Enhances the Biosynthesis of Phenolic Antioxidants in Fresh-Cut Carrot through a Synergistic Effect with Wounding. Molecules 22, 668.

Tang, D., Dong, Y., Ren, H., et al. (2014) A review of phytochemistry, metabolite changes, and medicinal uses of the common food mung bean and its sprouts (Vigna radiata). Chemistry Central Journal 8, 4-4.

Tang K, Zhan JC, Yang HR, et al. (2010) Changes of resveratrol and antioxidant enzymes during UV-induced plant defense response in peanut seedlings. J Plant Physiol 167, 95-102.

Thaipong, K., Boonprakob, U., Crosby, K., et al. (2006) Comparison of ABTS, DPPH, FRAP, and ORAC assays for estimating antioxidant activity from guava fruit extracts. Journal of Food Composition and Analysis 19, 669675.

Wang, H.B., Gui, M.Y., Tian, X., et al. (2017) Effects of UV-B on vitamin $\mathrm{C}$, phenolics, flavonoids and their related enzyme activities in mung bean sprouts (Vigna radiata). International Journal of Food Science and Technology 52, 827-833.

Wang, K.H., Lai, Y.H., Chang, J.C., et al. (2005) Germination of peanut kernels to enhance resveratrol biosynthesis and prepare sprouts as a functional vegetable. Journal of Agricultural and Food Chemistry 53, 242246.

Wang, M.L. and Pittman, R.N. (2009) Resveratrol content in seeds of peanut germplasm quantified by HPLC. Plant Genetic Resources 7, 80-83.
Yang, Q.Q., Cheng, L., Long, Z.Y., et al. (2019) Comparison of the Phenolic Profiles of Soaked and Germinated Peanut Cultivars via UPLC-QTOF-MS. Antioxidants (Basel) 8.

Yu, M., Liu, H.Z., Yang, Y., et al. (2016) Optimising germinated conditions to enhance yield of resveratrol content in peanut sprout using response surface methodology. International Journal of Food Science and Technology 51, 1754-1761.

Zhang, Q., Xiang, J., Zhang, L., et al. (2014) Optimizing soaking and germination conditions to improve gamma-aminobutyric acid content in japonica and indica germinated brown rice. Journal of Functional Foods 10, 283-291.

\section{Acknowledgements}

The authors greatly appreciate the financial support from the Vietnamese and Belgian cooperation program. The authors also thank to Central Lab of Food Science and Technology (CL-FST), Faculty of Food Science and Technology, VNUA for their support. The authors would like to thank MSc. Thi Hong Nguyen for her technical support and MSc. Thuy Huong Nguyen for her proofreading. 